\title{
Extended Soliton Solutions in an Effective Action for $S U(2)$ Yang-Mills Theory
}

\author{
Nobuyuki SAWADO, Noriko SHIIKI and Shingo TANAKA \\ Department of Physics, Faculty of Science and Technology, Tokyo University of Science, \\ Noda, Chiba 278-8510, Japan \\ E-mail:sawado@ph.noda.tus.ac.jp,norikoshiiki@mail.goo.ne.jp
}

Received October 25, 2005, in final form January 25, 2006; Published online January 31, 2006

Original article is available at http://www.emis.de/journals/SIGMA/2006/Paper016/

\begin{abstract}
The Skyrme-Faddeev-Niemi (SFN) model which is an $O(3) \sigma$ model in three dimensional space up to fourth-order in the first derivative is regarded as a low-energy effective theory of $S U(2)$ Yang-Mills theory. One can show from the Wilsonian renormalization group argument that the effective action of Yang-Mills theory recovers the SFN in the infrared region. However, the theory contains an additional fourth-order term which destabilizes the soliton solution. We apply the perturbative treatment to the second derivative term in order to exclude (or reduce) the ill behavior of the original action and show that the SFN model with the second derivative term possesses soliton solutions.
\end{abstract}

Key words: topological soliton; Yang-Mills theory; second derivative field theory

2000 Mathematics Subject Classification: 35Q51; 35G30; 70S15

\section{Introduction}

The Skyrme-Faddeev-Niemi (SFN) model which is an $O(3) \sigma$ model in three dimensional space up to fourth-order in the first derivative has topological soliton solutions with torus or knot-like structure. The model was initiated in 70's and the interest to it has been growing considerably. The numerical simulations were performed in $[1,2,3,4,5]$, the integrability was shown in [6], and the application to the condensed matter physics [7] and the Weinberg-Salam model [8] were also considered. The recent research especially focuses on the consistency between the SFN and fundamental theories such as QCD $[9,10,11,12]$. In those references, it is claimed that the SFN action should be induced from the $S U(2)$ Yang-Mills (YM) action at low energies. One can also show from the Wilsonian renormalization group argument that the effective action of YangMills theory recovers the SFN in the infrared region [13]. However, the derivative expansion for slowly varying fields $\boldsymbol{n}$ up to quartic order produces an additional fourth-order term in the SFN model, resulting in instability of the soliton solution.

Similar situations can be seen also in various topological soliton models. In the Skyrme model, the chirally invariant Lagrangian with quarks produces fourth order terms after the derivative expansion and they destabilize the soliton solution $[14,15]$. To recover the stability of the skyrmion, the author of [16] introduced a large number of higher order terms in the first derivative whose coefficients were determined from those of the Skyrme model by using the recursion relations. Alternatively, in [13] Gies pointed out the possibility that the second derivative order term can work as a stabilizer for the soliton.

In this paper, we examine the Gies's supposition by numerical analysis. In Section 2, we give an introduction to the Skyrme-Faddeev-Niemi model with its topological property. In Section 3, we show how to derive the SFN model action from the $S U(2)$ Yang-Mills theory. In Section 4, soliton solutions of this truncated YM action are studied. In order to find stable soliton solutions, 
we introduce a second derivative term which can be derived in a perturbative manner. The naive extremization scheme, however, produces the fourth order differential equation and the model has no stable soliton solution. In Section 5, the higher derivative theory and Ostrogradski's formulation are reviewed. We show the absence of bound state in the second derivative theory using an example in quantum mechanics and introduce the perturbative treatment for the second derivative theory. In Section 6, we present our numerical results. Section 7 contains concluding remarks.

\section{Skyrme-Faddeev-Niemi model}

The Faddeev-Niemi conjecture for the low-energy model of $S U(2)$ Yang-Mills theory is expressed by the effective action:

$$
S_{\mathrm{SFN}}=\Lambda^{2} \int d^{4} x\left[\frac{1}{2}\left(\partial_{\mu} \boldsymbol{n}\right)^{2}+\frac{g_{1}}{8}\left(\boldsymbol{n} \cdot \partial_{\mu} \boldsymbol{n} \times \partial_{\nu} \boldsymbol{n}\right)^{2}\right],
$$

where $\boldsymbol{n}(\boldsymbol{x})$ is a three component vector field normalized as $\boldsymbol{n} \cdot \boldsymbol{n}=1$. The mass scale parameter $\Lambda$ can be scaled out by the replacement $\Lambda x \rightarrow x$ and $\Lambda^{2} g_{1} \rightarrow g_{1}$, and for the static energy $E_{\text {stt }} / \Lambda \rightarrow E_{\text {stt. }}$. Stable soliton solutions exist when $g_{1}>0$.

The static field $\boldsymbol{n}(\boldsymbol{x})$ maps $\boldsymbol{n}: R^{3} \mapsto S^{2}$ and the configurations are classified by the topological maps characterized by a topological invariant $H$ called Hopf charge

$$
H=\frac{1}{32 \pi^{2}} \int A \wedge F, \quad F=d A
$$

where $F$ is the field strength and can be written as $F=(\boldsymbol{n} \cdot d \boldsymbol{n} \wedge d \boldsymbol{n})$.

The static energy $E_{\mathrm{stt}}$ from the action (1) has a topological lower bound [17],

$$
E_{\mathrm{stt}} \geq K H^{3 / 4}
$$

where $K=4 \sqrt{2} 3^{3 / 8} \pi^{2} \sqrt{g_{1}}$. Note that Ward improved this topological bound by using the Hopf map [18]. It seems, however, to be an upper bound of the model rather than lower bound.

Performing numerical simulation, one can find that the static configurations for $H=1,2$ have axial symmetry [3]. Thus "the toroidal ansatz" which was studied in [2] is suitable to be imposed on these configurations. The ansatz is given by

$$
\begin{aligned}
& n_{1}=\sqrt{1-w^{2}(\eta, \beta)} \cos (N \alpha+v(\eta, \beta)), \\
& n_{2}=\sqrt{1-w^{2}(\eta, \beta)} \sin (N \alpha+v(\eta, \beta)), \\
& n_{3}=w(\eta, \beta),
\end{aligned}
$$

where $(\eta, \beta, \alpha)$ are toroidal coordinates which are related to the $R^{3}$ as follows:

$$
x=\frac{a \sinh \eta \cos \alpha}{\tau}, \quad y=\frac{a \sinh \eta \sin \alpha}{\tau}, \quad z=\frac{a \sin \beta}{\tau}
$$

with $\tau=\cosh \eta-\cos \beta$.

The function $w(\eta, \beta)$ is subject to the boundary conditions $w(0, \beta)=1, w(\infty, \beta)=-1$ and is periodic in $\beta . v(\eta, \beta)$ is set to be $v(\eta, \beta)=M \beta+v_{0}(\eta, \beta)$ and $v_{0}(, \beta)$ is considered as a constant map. Equation (2) then gives $H=N M$.

In this paper we adopt a simpler ansatz than (4), which is defined by

$$
n_{1}=\sqrt{1-w^{2}(\eta)} \cos (N \alpha+M \beta),
$$




$$
\begin{aligned}
& n_{2}=\sqrt{1-w^{2}(\eta)} \sin (N \alpha+M \beta), \\
& n_{3}=w(\eta),
\end{aligned}
$$

where $w(\eta)$ satisfies the boundary conditions $w(0)=1, w(\infty)=-1$. We numerically study soliton solutions for both ansatz (4) and (5). By comparing those results, we find that this simple ansatz produces at most $10 \%$ errors and does not much effect to the property of the soliton solution.

By using (5), the static energy is written in terms of the function $w(\eta)$ as

$$
\begin{aligned}
& E_{\mathrm{stt}}=2 \pi^{2} a \int d \eta\left[\frac{\left(w^{\prime}\right)^{2}}{1-w^{2}}+\left(1-w^{2}\right) U_{M, N}(\eta)+\frac{g_{1}}{4 a^{2}} \sinh \eta \cosh \eta\left(w^{\prime}\right)^{2} U_{M, N}(\eta)\right], \\
& w^{\prime} \equiv \frac{d w}{d \eta}, \quad U_{M, N}(\eta) \equiv\left(M^{2}+\frac{N^{2}}{\sinh ^{2} \eta}\right) .
\end{aligned}
$$

The Euler-Lagrange equation of motion is then derived as

$$
\begin{gathered}
\frac{w^{\prime \prime}}{1-w^{2}}+\frac{w w^{\prime 2}}{\left(1-w^{2}\right)^{2}}+U_{M, N}(\eta) w+\frac{g_{1}}{2 a^{2}}\left(-2 N^{2} \operatorname{coth}^{2} \eta w^{\prime} U_{M, N}(\eta) w^{\prime}\right. \\
\left.+\left(\cosh ^{2} \eta+\sinh ^{2} \eta\right)+\sinh \eta \cosh \eta U_{M, N}(\eta) w^{\prime \prime}\right)=0 .
\end{gathered}
$$

The variation with respect to $a$ produces the equation for variable $a$. Soliton solutions are obtained by solving the equations for $a$ as well as for $w$.

\section{Effective action in the Yang-Mills theory with CFNS decomposition}

In this section, we briefly review how to derive the SFN effective action from the action of $\mathrm{SU}(2)$ Yang-Mills theory in the infrared limit $[11,13]$. For the gauge fields $\boldsymbol{A}_{\mu}$, the Cho-FaddeevNiemi-Shabanov decomposition is applied [9, 10, 11, 12]

$$
\boldsymbol{A}_{\mu}=\boldsymbol{n} C_{\mu}+\left(\partial_{\mu} \boldsymbol{n}\right) \times \boldsymbol{n}+\boldsymbol{W}_{\mu} .
$$

The first two terms are the "electric" and "magnetic" Abelian connection, and $\boldsymbol{W}_{\mu}$ are chosen to be orthogonal to $\boldsymbol{n}$, i.e. $\boldsymbol{W}_{\mu} \cdot \boldsymbol{n}=0$. Obviously, the degrees of freedom on the left- and right-hand side of equation (7) do not match. While the LHS describes $3_{\text {color }} \times 4_{\text {Lorentz }}=12$, the RHS is comprised of $\left(C_{\mu}:\right) 4_{\text {Lorentz }}+(\boldsymbol{n}:) 2_{\text {color }}+\left(\boldsymbol{W}_{\mu}:\right) 3_{\text {color }} \times 4_{\text {Lorentz }}-4_{\boldsymbol{n} \cdot \boldsymbol{W}_{\mu}=0}=14$ degrees freedom. Shabanov introduced in his paper [11] the following constraint

$$
\chi\left(\boldsymbol{n}, C_{\mu}, \boldsymbol{W}_{\mu}\right)=0, \quad \text { with } \quad \chi \cdot \boldsymbol{n}=0 .
$$

The generating functional of YM theory can be written by using equation (7) as

$$
\mathcal{Z}=\int \mathcal{D} \boldsymbol{n} \mathcal{D} C \mathcal{D} \boldsymbol{W} \delta(\chi) \Delta_{\mathrm{FP}} \Delta_{\mathrm{S}} e^{-S_{\mathrm{YM}}-S_{\mathrm{gf}}}
$$

$\Delta_{\mathrm{FP}}$ and $S_{\mathrm{gf}}$ are the Faddeev-Popov determinant and the gauge fixing action respectively, and Shabanov introduced another determinant $\Delta_{\mathrm{S}}$ corresponding to the condition $\chi=0$. YM and the gauge fixing action is given by

$$
S_{\mathrm{YM}}+S_{\mathrm{gf}}=\int d^{4} x\left[\frac{1}{4 g^{2}} \boldsymbol{F}_{\mu \nu} \cdot \boldsymbol{F}_{\mu \nu}+\frac{1}{2 \alpha_{\mathrm{g}} g^{2}}\left(\partial_{\mu} \boldsymbol{A}_{\mu}\right)^{2}\right] .
$$


Inserting equation (7) into the action, one obtains the form:

$$
\begin{aligned}
\mathcal{Z}=\int \mathcal{D} \boldsymbol{n} e^{-\mathcal{S}_{\text {eff }}(\boldsymbol{n})}= & \int \mathcal{D} \boldsymbol{n} e^{-\mathcal{S}_{\mathrm{cl}}(\boldsymbol{n})} \int \mathcal{D} \tilde{C} \mathcal{D} \boldsymbol{W}_{\mu} \Delta_{\mathrm{FP}} \Delta_{\mathrm{S}} \delta(\boldsymbol{\chi}) \\
& \times e^{-\left(1 / 2 g^{2}\right) \int\left(\tilde{C}_{\mu} M_{\mu \nu}^{C} \tilde{C}_{\nu}+\boldsymbol{W}_{\mu} \bar{M}_{\mu \nu}^{W} \boldsymbol{W}_{\nu}+2 C_{\nu} K_{\nu}^{C}+2 \boldsymbol{W}_{\mu} \cdot \boldsymbol{K}_{\mu}^{W}\right)}
\end{aligned}
$$

with

$$
\begin{aligned}
& M_{\mu \nu}^{C}=-\partial^{2} \delta_{\mu \nu}+\partial_{\mu} \boldsymbol{n} \cdot \partial_{\nu} \boldsymbol{n}, \\
& M_{\mu \nu}^{\boldsymbol{W}}=-\partial^{2} \delta_{\mu \nu}-\partial_{\mu} \boldsymbol{n} \otimes \partial_{\nu} \boldsymbol{n}+\partial_{\nu} \boldsymbol{n} \otimes \partial_{\mu} \boldsymbol{n}, \\
& \boldsymbol{Q}_{\mu \nu}^{C}=\partial_{\mu} \boldsymbol{n} \partial_{\nu}+\partial_{\nu} \boldsymbol{n} \partial_{\mu}+\partial_{\mu} \partial_{\nu} \boldsymbol{n}, \\
& K_{\mu \nu}^{C}=\partial_{\nu}\left(\boldsymbol{n} \cdot \partial_{\nu} \boldsymbol{n} \times \partial_{\mu} \boldsymbol{n}\right)+\partial_{\mu} \boldsymbol{n} \cdot \partial^{2} \boldsymbol{n} \times \boldsymbol{n}, \\
& \boldsymbol{K}_{\mu \nu}^{\boldsymbol{W}}=\partial_{\mu}\left(\boldsymbol{n} \times \partial^{2} \boldsymbol{n}\right), \quad\left(\text { in gauge } \alpha_{\mathrm{g}}=1\right)
\end{aligned}
$$

and

$$
\begin{aligned}
& \bar{M}_{\mu \nu}^{\boldsymbol{W}}:=M_{\mu \nu}^{\boldsymbol{W}}+\tilde{\boldsymbol{Q}}_{\mu s}^{C} M_{s \lambda}^{C-1} \boldsymbol{Q}_{\lambda \nu}^{C}, \\
& \tilde{C}_{\mu}=C_{\mu}+\boldsymbol{W}_{s} \cdot \boldsymbol{Q}_{s \lambda} M_{\lambda \mu}^{C-1} .
\end{aligned}
$$

Here, $\tilde{\boldsymbol{Q}}_{\mu s}^{C}$ has same form of $\boldsymbol{Q}_{\mu s}^{C}$ but differentiates to the right objects of $M_{s \lambda}^{C-1} \boldsymbol{Q}_{\lambda \nu}^{C}$. The classical action of $\boldsymbol{n}$ including the gauge fixing term is given by

$$
\mathcal{S}_{\mathrm{cl}}=\int d^{4} x\left[\frac{1}{4 g^{2}}\left(\partial_{\mu} \boldsymbol{n} \times \partial_{\nu} \boldsymbol{n}\right)^{2}+\frac{1}{2 \alpha_{\mathrm{g}} g^{2}}\left(\partial^{2} \boldsymbol{n} \times \boldsymbol{n}\right)^{2}\right] .
$$

The $\delta$ functional is expressed by its Fourier transform

$$
\delta(\boldsymbol{\chi})=\int \mathcal{D} \phi e^{-i \int\left(\boldsymbol{\phi} \cdot \partial \boldsymbol{W}_{\mu}+\boldsymbol{\phi} \cdot C_{\mu} \boldsymbol{n} \times \boldsymbol{W}_{\mu}+(\boldsymbol{\phi} \cdot \boldsymbol{n})\left(\partial_{\mu} \boldsymbol{n} \cdot \boldsymbol{W}_{\mu}\right)\right)} .
$$

Integrating over $C, \boldsymbol{W}, \boldsymbol{\phi}$, we finally obtain

$$
e^{-S_{\mathrm{eff}}}=e^{-S_{\mathrm{cl}}} \Delta_{\mathrm{FP}} \Delta_{\mathrm{S}}\left(\operatorname{det} M^{C}\right)^{-1 / 2}\left(\operatorname{det} \bar{M}^{\boldsymbol{W}}\right)^{-1 / 2}\left(\operatorname{det}-\tilde{Q}_{\mu}^{\phi}\left(\bar{M}^{\boldsymbol{W}}\right)_{\mu \nu}^{-1} Q_{\nu}^{\phi}\right)^{-1 / 2},
$$

where $Q_{\nu}^{\phi}=i\left(-\partial_{\mu}+\partial_{\mu} \boldsymbol{n} \otimes \boldsymbol{n}\right)$ and $\tilde{Q}_{\mu}^{\phi}$ differentiates to the right. We perform the derivative expansion for the four determinants in equation (8) under the following assumptions

(i) the theory is valid for the momenta $p$ with $k<p<\Lambda(k, \Lambda$ are infrared and ultraviolet cut-off),

(ii) $|\partial \boldsymbol{n}| \ll k$,

(iii) the higher derivative terms, such as $\partial^{2} \boldsymbol{n}$ are omitted.

The effective action is then given by

$$
S_{\text {eff }}=\int d^{4} x\left[\frac{1}{2}\left(\partial_{\mu} \boldsymbol{n}\right)^{2}+\frac{g_{1}}{8}\left(\partial_{\mu} \boldsymbol{n} \times \partial_{\nu} \boldsymbol{n}\right)^{2}+\frac{g_{2}}{8}\left(\partial_{\mu} \boldsymbol{n}\right)^{4}\right] .
$$

For $g_{1}>0$ and $g_{2}=0$, the action is identical to the FSN effective action (1).

In order to get the stable soliton solutions, $g_{2}$ must be positive [2]. However, $g_{2}$ is found to be negative according to the above analysis (see [13]). Therefore we consider higher-derivative terms and investigate if the model with the higher-derivatives possess soliton solutions. 


\section{Search for the stable soliton solutions (1)}

The static energy is derived from equation (9) as

$$
\begin{aligned}
E_{\mathrm{stt}} & =\int d^{3} x\left[\frac{1}{2}\left(\partial_{i} \boldsymbol{n}\right)^{2}+\frac{g_{1}}{8}\left(\partial_{i} \boldsymbol{n} \times \partial_{j} \boldsymbol{n}\right)^{2}+\frac{g_{2}}{8}\left(\partial_{i} \boldsymbol{n}\right)^{4}\right] \\
& :=E_{2}(\boldsymbol{n})+E_{4}^{(1)}(\boldsymbol{n})+E_{4}^{(2)}(\boldsymbol{n}) .
\end{aligned}
$$

A spatial scaling behavior of the static energy, so called Derrick's scaling argument, can be applied to examine the stability of the soliton [19]. Considering the map $\boldsymbol{x} \mapsto \boldsymbol{x}^{\prime}=\mu \boldsymbol{x}(\mu>0)$, with $\boldsymbol{n}^{(\mu)} \equiv \boldsymbol{n}(\mu \boldsymbol{x})$, the static energy scales as

$$
\begin{aligned}
e(\mu) & =E_{\text {stt }}\left(\boldsymbol{n}^{(\mu)}\right) \\
& =E_{2}\left(\boldsymbol{n}^{(\mu)}\right)+E_{4}^{(1)}\left(\boldsymbol{n}^{(\mu)}\right)+E_{4}^{(2)}\left(\boldsymbol{n}^{(\mu)}\right) \\
& =\frac{1}{\mu} E_{2}(\boldsymbol{n})+\mu\left(E_{4}^{(1)}(\boldsymbol{n})+E_{4}^{(2)}(\boldsymbol{n})\right) .
\end{aligned}
$$

Derrick's theorem states that if the function $e(\mu)$ has no stationary point, the theory has no static solutions of the field equation with finite density, other than the vacuum. Conversely, if $e(\mu)$ has stationary point, the possibility of having finite energy soliton solutions is not excluded. equation (10) is stationary at $\mu=\sqrt{E_{2} /\left(E_{4}^{(1)}+E_{4}^{(2)}\right)}$. Then, the following inequality

$$
\begin{aligned}
g_{1}\left(\partial_{i} \boldsymbol{n}\right. & \left.\times \partial_{j} \boldsymbol{n}\right)^{2}+g_{2}\left(\partial_{i} \boldsymbol{n}\right)^{2}\left(\partial_{j} \boldsymbol{n}\right)^{2} \\
& =g_{1}\left(\partial_{i} \boldsymbol{n}\right)^{2}\left(\partial_{j} \boldsymbol{n}\right)^{2}-g_{1}\left(\partial_{i} \boldsymbol{n} \cdot \partial_{j} \boldsymbol{n}\right)^{2}+g_{2}\left(\partial_{i} \boldsymbol{n}\right)^{2}\left(\partial_{j} \boldsymbol{n}\right)^{2} \\
& \geqq g_{2}\left(\partial_{i} \boldsymbol{n} \cdot \partial_{j} \boldsymbol{n}\right)^{2} \quad\left(\because\left(\partial_{i} \boldsymbol{n}\right)^{2}\left(\partial_{j} \boldsymbol{n}\right)^{2} \geqq\left(\partial_{i} \boldsymbol{n} \cdot \partial_{j} \boldsymbol{n}\right)^{2}\right)
\end{aligned}
$$

ensures the possibility of existence of the stable soliton solutions for $g_{2} \geqq 0$. As mentioned in the Section $3, g_{2}$ should be negative at least within our derivative expansion analysis of YM theory.

A promising idea to tackle the problem was suggested by Gies [13]. He considered the following type of effective action, accompanying second derivative term

$$
S_{\text {eff }}=\int d^{4} x\left[\frac{1}{2}\left(\partial_{\mu} \boldsymbol{n}\right)^{2}+\frac{g_{1}}{8}\left(\partial_{\mu} \boldsymbol{n} \times \partial_{\nu} \boldsymbol{n}\right)^{2}-\frac{g_{2}}{8}\left(\partial_{\mu} \boldsymbol{n}\right)^{4}+\frac{g_{2}}{8}\left(\partial^{2} \boldsymbol{n} \cdot \partial^{2} \boldsymbol{n}\right)\right] .
$$

Here we choose positive value of $g_{2}$ and assign the explicit negative sign to the third term. In principle, it is possible to estimate the second derivative term by the derivative expansion without neglecting throughout the calculation.

The static energy of equation (11) with the ansatz (5) is written as

$$
\begin{aligned}
E_{\mathrm{stt}}= & 2 \pi^{2} a \int d \eta\left[\frac{\left(w^{\prime}\right)^{2}}{1-w^{2}}+\left(1-w^{2}\right) U_{M, N}(\eta)+\frac{g_{1}}{4 a^{2}} \sinh \eta \cosh \eta\left(w^{\prime}\right)^{2} U_{M, N}(\eta)\right. \\
& +\frac{g_{2}}{4 a^{2}}\left[-\sinh \eta \cosh \eta\left[\frac{\left(w^{\prime}\right)^{2}}{1-w^{2}}+\left(1-w^{2}\right) U_{M, N}(\eta)\right]^{2}\right. \\
& +\left(\operatorname{coth} \eta+\sinh ^{2} \eta-\sinh \eta \cosh \eta\right) \frac{\left(w^{\prime}\right)^{2}}{1-w^{2}} \\
& +\left(\sinh \eta \cosh \eta-\sinh ^{2} \eta\right)\left(1-w^{2}\right) M^{2}+2\left\{\frac{w\left(w^{\prime}\right)^{3}}{\left(1-w^{2}\right)^{2}}+\frac{w^{\prime} w^{\prime \prime}}{1-w^{2}}+w w^{\prime} U_{M, N}(\eta)\right\} \\
& \left.\left.+\sinh \eta \cosh \eta\left\{\frac{1}{1-w^{2}}\left[\frac{\left(w^{\prime}\right)^{2}}{1-w^{2}}+w w^{\prime \prime}+\left(1-w^{2}\right) U_{M, N}(\eta)\right]^{2}+\left(w^{\prime \prime}\right)^{2}\right\}\right]\right]
\end{aligned}
$$


where $w^{\prime \prime} \equiv \frac{d^{2} w}{d \eta^{2}}$. The Euler-Lagrange equation of motion is derived by

$$
-\frac{d^{2}}{d \eta^{2}}\left(\frac{\partial E_{\mathrm{stt}}}{\partial w^{\prime \prime}}\right)+\frac{d}{d \eta}\left(\frac{\partial E_{\mathrm{stt}}}{\partial w^{\prime}}\right)-\frac{\partial E_{\mathrm{stt}}}{\partial w}=0
$$

which is too complicated to write down explicitly and hence we adopt the following notation

$$
f_{0}\left(w, w^{\prime}, w^{\prime \prime}\right)+g_{1} f_{1}\left(w, w^{\prime}, w^{\prime \prime}\right)+g_{2} f_{2}\left(w, w^{\prime}, w^{\prime \prime}, w^{(3)}, w^{(4)}\right)=0 .
$$

Here $w^{(3)}, w^{(4)}$ represent the third and the fourth derivative with respect to $\eta$. The first two terms of equation (12) are identical to those in equation (6). Unfortunately, we could not find out stable soliton solutions from equation (12) for any (even in the quite small) value of $g_{2}$.

From the relation

$$
\int d^{4} x\left[\left(\partial^{2} \boldsymbol{n} \cdot \partial^{2} \boldsymbol{n}\right)-\left(\partial_{\mu} \boldsymbol{n}\right)^{4}\right]=\int d^{4} x\left(\partial^{2} \boldsymbol{n} \times \boldsymbol{n}\right)^{2},
$$

one easily finds that the static energy obtained from the last two terms in equation (11)

$$
\tilde{E}_{4}^{(2)}=\int d^{3} x\left(\partial^{2} \boldsymbol{n} \times \boldsymbol{n}\right)^{2}
$$

gives the positive contribution. The total static energy is stationary at $\mu=\sqrt{E_{2} /\left(E_{4}^{(1)}+\tilde{E}_{4}^{(2)}\right)}$ and hence the possibility of existence of soliton solutions is not excluded. And also, the positivity of equation (13) does not spoil the lower bound (3) of original SFN and the possibility still remains.

The standard action (1) has a fourth differential order term, but only quadratic in time derivative. On the other hand, the action (11) involves a term with fourth order in time derivative. Then, even if the static energy is constructed only by the positive terms, it is in general not bounded from below. As a result, the stability of the soliton is unclear. In the next section, we report the basic feature of the higher derivative action and introduce the method to avoid the problems inherent by applying an example in quantum mechanics.

\section{$5 \quad$ Higher derivative theory}

In this section, we make a small detour, i.e., we review the problems in the higher derivative theory [20, 21, 22, 23, 24] which essentially falls into two categories. The first problem concerns the increase in the number of degrees of freedom. For example, if the theory contains second derivative terms, the equation of motion becomes up to the order in the fourth derivative. Thus, four parameters are required for the initial conditions. If one considers higher-order terms, the situation gets worse. However, this is not a serious problem for our study because our concern is existence of static soliton solutions. The second problem is that the actions of the theory are not bounded from below. This feature makes the higher derivative theories unstable.

We briefly review the Lagrangian and the Hamiltonian formalism with higher derivative called the Ostrogradski method. We consider the Lagrangian containing up to $n$th order derivatives

$$
S=\int d t \mathcal{L}\left(q, \dot{q}, \ldots, q^{(n)}\right) .
$$

Taking the variation of the action $\delta S=0$ leads the Euler-Lagrange equation of motion

$$
\sum_{i=0}^{n}(-1)^{i} \frac{d^{i}}{d t^{i}}\left(\frac{\partial \mathcal{L}}{\partial q^{(i)}}\right)=0 .
$$


The Hamiltonian is obtained by introducing $n$ generalized momenta

$$
p_{i}=\sum_{j=i+1}^{n}(-1)^{j-i-1} \frac{d^{j-i-1}}{d t^{j-i-1}}\left(\frac{\partial \mathcal{L}}{\partial q^{(j)}}\right), \quad i=1, \ldots, n,
$$

or

$$
p_{n}=\frac{\partial \mathcal{L}}{\partial q^{(n)}}, \quad p_{i}=\frac{\partial \mathcal{L}}{\partial q^{(i)}}-\frac{d}{d t} p_{i+1}, \quad i=1, \ldots, n-1
$$

and $n$ independent variables

$$
q_{1} \equiv q, \quad q_{i} \equiv q^{(i-1)}, \quad i=2, \ldots, n
$$

The Lagrangian now depends on the $n$ coordinates $q_{i}$ and on the first derivative $\dot{q}_{n}=q^{(n)}$. The Hamiltonian is defined as

$$
\mathcal{H}\left(q_{i}, p_{i}\right)=\sum_{i=1}^{n} p_{i} \dot{q}_{i}-\mathcal{L}=\sum_{i=1}^{n-1} p_{i} q_{i+1}+p_{n} \dot{q}_{n}-\mathcal{L} .
$$

The canonical equations of motion turn out to be

$$
\dot{q}_{i}=\frac{\partial \mathcal{H}}{\partial p_{i}}, \quad \dot{p}_{i}=-\frac{\partial \mathcal{H}}{\partial q_{i}} .
$$

Thus, we replace a theory of one coordinate $q$ system obeying $2 n$-th differential equation with a set of 1 -st order canonical equations for $2 n$ phase-space variables $\left[q_{i}, p_{i}\right]$.

We consider a simple example including second derivative term [24], defined as

$$
\mathcal{L}=\frac{1}{2}\left(1+\varepsilon^{2} \omega^{2}\right) \dot{q}^{2}-\frac{1}{2} \omega^{2} q^{2}-\frac{1}{2} \varepsilon^{2} \ddot{q}^{2}
$$

where constant $\epsilon$ works as a coupling constant of second derivative term. The equation of motion is

$$
\left(1+\varepsilon^{2} \omega^{2}\right) \ddot{q}+\omega^{2} q+\varepsilon^{2} q^{(4)}=0 .
$$

From equation (14), one gets

$$
\pi_{\dot{q}}=\frac{\partial \mathcal{L}}{\partial \ddot{q}}=-\varepsilon^{2} \ddot{q}, \quad \pi_{q}=\frac{\partial \mathcal{L}}{\partial \dot{q}}-\frac{d}{d t}\left(\frac{\partial \mathcal{L}}{\partial \ddot{q}}\right)=\left(1+\varepsilon^{2} \omega^{2}\right) \dot{q}+\varepsilon^{2} \dddot{q} .
$$

Thus the Hamiltonian becomes

$$
\begin{aligned}
\mathcal{H} & =\dot{q} \pi_{q}+\ddot{q} \pi_{\dot{q}}-\mathcal{L} \\
& =\dot{q} \pi_{q}-\frac{1}{2 \varepsilon^{2}} \pi_{\dot{q}}^{2}-\frac{1}{2}\left(1+\varepsilon^{2} \omega^{2}\right) \dot{q}^{2}+\frac{1}{2} \omega^{2} q^{2} .
\end{aligned}
$$

We introduce the new canonical variables

$$
\begin{aligned}
& q_{+}=\frac{1}{\omega \sqrt{1-\varepsilon^{2} \omega^{2}}}\left(\varepsilon^{2} \omega^{2} \dot{q}-\pi_{q}\right), \quad p_{+}=\frac{w}{\sqrt{1-\varepsilon^{2} \omega^{2}}}\left(q-\pi_{\dot{q}}\right), \\
& q_{-}=\frac{\varepsilon}{\sqrt{1-\varepsilon^{2} \omega^{2}}}\left(\dot{q}-\pi_{q}\right), \quad p_{-}=\frac{1}{\varepsilon \sqrt{1-\varepsilon^{2} \omega^{2}}}\left(\varepsilon^{2} \omega^{2} q-\pi_{\dot{q}}\right),
\end{aligned}
$$


and the Hamiltonian will have the following form by using these variables

$$
\mathcal{H} \rightarrow \frac{1}{2}\left(p_{+}^{2}+\omega^{2} q_{+}^{2}\right)-\frac{1}{2}\left(p_{-}^{2}+\frac{1}{\varepsilon^{2}} q_{-}^{2}\right)
$$

The corresponding energy spectra is then given by

$$
E=\left(n+\frac{1}{2}\right) \omega-\left(m+\frac{1}{2}\right) \frac{1}{\varepsilon}, \quad n, m=0,1,2, \ldots
$$

One can see that in the limit $\epsilon \rightarrow 0$ the energy goes to negative infinity rather than approaching to the harmonic oscillator energy eigenstates.

To obtain physically meaningful solutions, we employ the perturbative analysis where the solution is expanded in terms of the small coupling constant and the Euler-Lagrange equation of motion is replaced with the corresponding perturbative equation. The solutions of the equations of motion that are ill behaved in the limit $\epsilon \rightarrow 0$ are excluded from the very beginning [22, 23, 24].

We assume that the solution of equation (15) can be written as

$$
q_{\text {pert }}(t)=\sum_{n=0}^{\infty} \epsilon^{n} q(t) .
$$

Substituting equation (17) into equation (15) and taking time derivatives of these equations, we obtain the constraints for higher derivative terms

$$
\begin{aligned}
& O\left(\epsilon^{0}\right) \\
& \text { equation : } \ddot{q}_{0}+\omega^{2} q_{0}=0, \\
& \text { constraints : } \quad \dddot{q}_{0}=-\omega^{2} \dot{q}_{0}, \quad \dddot{q}_{0}=\omega^{4} q_{0} . \\
& O\left(\epsilon^{2}\right) \\
& \text { equation : } \quad \ddot{q}_{2}+\omega^{2} \ddot{q}_{0}+\omega^{2} q_{2}+\dddot{q}_{0}=0 \text {, } \\
& \Rightarrow \ddot{q}_{2}+\omega^{2} q_{2}=0, \quad(\text { using (18)), } \\
& \text { constraints : } \quad \dddot{q}_{2}=-\omega^{2} \dot{q}_{2}, \quad \dddot{q}_{2}=\omega^{4} q_{2} . \\
& O\left(\epsilon^{4}\right) \\
& \text { equation : } \quad \ddot{q}_{4}+\omega^{2} \ddot{q}_{2}+\omega^{2} q_{4}+\dddot{q}_{2}=0 \text {, } \\
& \Rightarrow \ddot{q}_{4}+\omega^{2} q_{4}=0, \quad \text { (using (19)), } \\
& \text { constraints : } \quad \dddot{q}_{4}=-\omega^{2} \dot{q}_{4}, \quad \dddot{q}_{4}=\omega^{4} q_{4} \text {. }
\end{aligned}
$$

Combining these results, we find the perturbative equation of motion up to $O\left(\epsilon^{4}\right)$

$$
\ddot{q}_{\text {pert }}+\omega^{2} q_{\text {pert }}=O\left(\epsilon^{6}\right)
$$

which is the equation for harmonic oscillator.

This perturbative method can successfully exclude the ill behavior of the second derivative theory. Of course our problem is different from this quantum mechanics example. While the energy spectra (16) is quantum value, the energy of the soliton is static and classical. Nevertheless, the perturbative method may give some hint to tackle our problem. In the next section, we will apply the method to the action with the second derivative term (11) and explore the stable soliton solutions. 


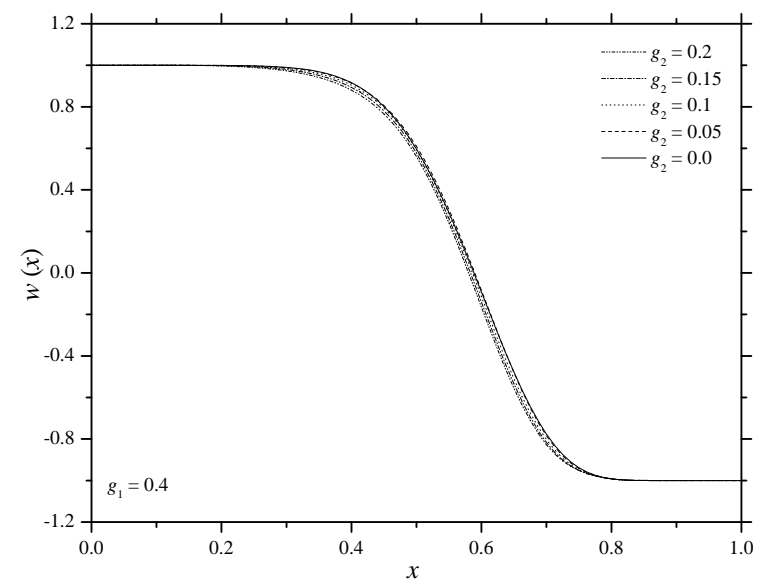

Figure 1. The function $w(\eta)$ for $g_{1}=0.4$, $g_{2}=0,0.05,0.1,0.15,0.2$ (the rescaling radial coordinate $x=\eta /(1-\eta)$ is used $)$.

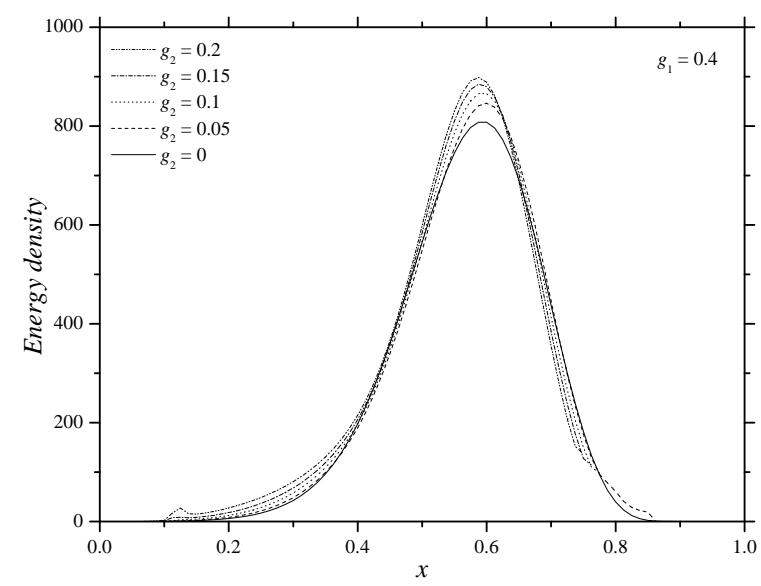

Figure 2. The energy density for $g_{1}=0.4$, $g_{2}=0,0.05,0.1,0.15,0.2$.

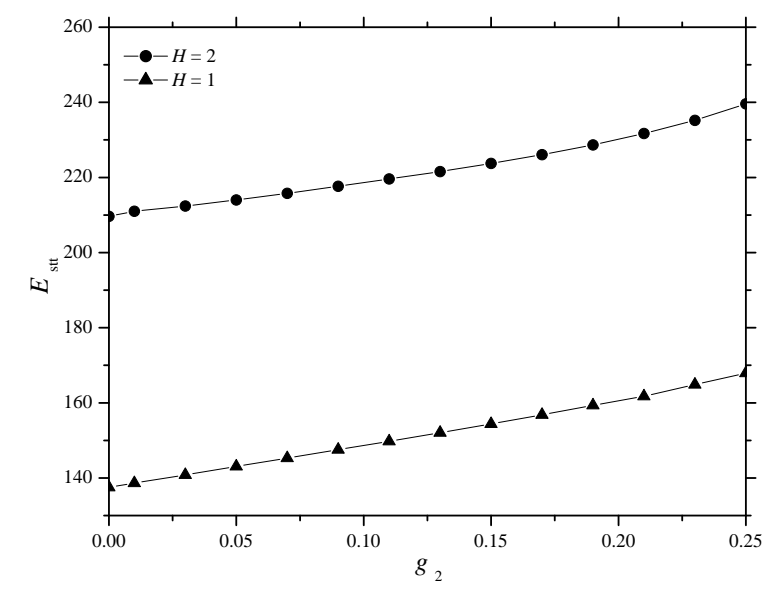

Figure 3. The energy as a function of $g_{2}\left(g_{1}=0.4\right)$.

\section{Search for the stable soliton solutions (2) (perturbative expansion method)}

As in the case of quantum mechanics, we assume that $g_{2}$ is relatively small and can be considered as a perturbative coupling constant. Thus, in analogy of (17), the perturbative solution is written by a power series in $g_{2}$

$$
w(\eta)=\sum_{n=0}^{\infty} g_{2}^{n} w_{n}(\eta) .
$$

Substituting equation (20) into equation (12), we obtain the classical field equation in $O\left(g_{2}^{0}\right)$

$$
f_{0}\left(w_{0}, w_{0}^{\prime}, w_{0}^{\prime \prime}\right)+g_{1} f_{1}\left(w_{0}, w_{0}^{\prime}, w_{0}^{\prime \prime}\right)=0 .
$$

Taking derivatives for both sides in equation (21) and solving for $w_{0}^{\prime \prime}$, we obtain the following form of the constraint equations for higher derivatives

$$
w_{0}^{(i)}=F^{(i)}\left(w_{0}, w_{0}^{\prime}\right), \quad i=2,3,4 .
$$

The equation in $O\left(g_{2}^{1}\right)$ can be written as

$$
\left(f_{0}+g_{2} f_{1}\right)_{O\left(g_{2}^{1}\right)}+f_{2}\left(w_{0}, w_{0}^{\prime}, w_{0}^{\prime \prime}, w_{0}^{(3)}, w_{0}^{(4)}\right)=0 .
$$


Substituting the constraint equations (22) into equation (23) and eliminating the higher derivative terms, one can obtain the perturbative equation of motion

$$
f_{0}\left(w, w^{\prime}, w^{\prime \prime}\right)+g_{1} f_{1}\left(w, w^{\prime}, w^{\prime \prime}\right)+g_{2} \tilde{f}_{2}\left(w, w^{\prime}\right)=O\left(g_{2}^{2}\right) .
$$

Now equation (24) has stable soliton solutions.

Our results of the estimated function $w(\eta)$ and the energy density are displayed in Figs. 1 and 2. (In all figures, we show the results for the case of Hopf charge $H=2 ; N=2, M=1$.) The dependence of the total energy on $g_{2}$ is shown in Fig. 3. The change is moderate with respect to $g_{2}$.

\section{Summary}

In this paper we have studied the Skyrme-Faddeev-Niemi model and its extensions by introducing the reduction scheme of the $S U(2)$ Yang-Mills theory to the corresponding low-energy effective model. The requirement of consistency between the low-energy effective action of the YM and the SFN type model leads us to take into account second derivative terms in the action. However, we found that such an action including the second derivative terms does not have stable soliton solutions. This is due to the absence of the energy bound in higher derivative theory. This fact inspired us to employ the perturbative analysis to our effective action. Within the perturbative analysis, we were able to obtain the stable soliton solutions.

Our analysis is based on perturbation and the coupling constant $g_{2}$ is assumed to be small. However, Wilsonian renormalization analysis of YM theory [13] suggests that the coupling constants $g_{1}, g_{2}$ (and the mass scale parameter $\Lambda$ ) depend on the renormalization group time $t=\log k / \Lambda$ ( $k, \Lambda$ are infrared, ultraviolet cutoff parameter $)$ and those are almost comparable. To improve the analysis, we could perform the next order of perturbation, but it is tedious and spoils the simplicity of the SFN model.

It should be noted that our solutions do not differ much from the solution of original SFN model, at least in the perturbative regime. We suspect that an appropriate truncation (such as "extra fourth order term + second derivative term") always supplies the stable solutions that are close to the original SFN model. Thus we conclude that the topological soliton model comprised of the "kinetic term + a special fourth order term" like SFN model is a good approximation.

Since our results were obtained from numerical study in the perturbative approach, it is uncertain whether the existence of the soliton is kept for larger coupling constant $g_{2}$. To confirm that, we should proceed to investigate next order perturbation, or, otherwise, find some analytical evidence for that. We point out that the perturbative treatment is only used for excluding the ill behavior of the second derivative field theory. We hope that applying this prescription does not alter the essential feature of the solutions.

Finally, let us mention the application of the soliton solutions to the glueball. Obviously this is one of the main interests to study the model and, many authors have given discussions on this subject $[13,25,26]$. On the other hand, the possibility of the magnetic condensation of the QCD vacuum within the Cho-Faddeev-Niemi-Shabanov decomposed Yang-Mills theory have been studied by Kondo [27]. The author claims the existence of nonzero off diagonal gluon mass $M_{X}$, which is induced in terms of the condensation of the magnetic potential part of the decomposition $\boldsymbol{B}_{\mu} \sim\left(\partial_{\mu} \boldsymbol{n}\right) \times \boldsymbol{n}$, as

$$
M_{X}^{2}=\left\langle\boldsymbol{B}_{\mu} \cdot \boldsymbol{B}_{\mu}\right\rangle=\left\langle\left(\partial_{\mu} \boldsymbol{n}\right)^{2}\right\rangle \text {. }
$$

Throughout our calculation, we scaled out the mass scale parameter $\Lambda$ in the action (1) but, in this sense, it should reflect the information of such gluon mass, or the condensation property of the vacuum. After a careful examination of the value of $\Lambda$, we will be able to make a prediction for the glueball mass. 


\section{Acknowledgements}

We are grateful to Kei-Ichi Kondo for drawing our attention to this subject and for many useful advises. We also thank M. Hirayama and J. Yamashita for valuable discussions.

[1] Faddeev L., Niemi A., Knots and particles, Nature, 1997, V.387, 58-61; hep-th/9610193.

[2] Gladikowski J., Hellmund M., Static solitons with nonzero hopf number, Phys. Rev. D, 1997, V.56, 51945199; hep-th/9609035.

[3] Battye R.A., Sutcliffe P.M., Solitons, links and knots, Proc. R. Soc. Lond. Ser. A Math. Phys. Eng. Sci., 1999, V.455, 4301-4331; hep-th/9811077.

[4] Hietarinta J., Salo P., Faddeev-Hopf knots: dynamics of linked unknots, Phys. Lett. B, 1999, V.451, 60-67; hep-th/9811053.

[5] Hietarinta J., Salo P., Ground state in the Faddeev-Skyrme model, Phys. Rev. D, 2000, V.62, 081701, 4 pages.

[6] Aratyn H., Ferreira L.A., Zimerman A.H., Toroidal solitons in (3+1)-dimensional integrable theories, Phys. Lett. B, 1999, V.456, 162-170; hep-th/9902141.

[7] Babaev E., Faddeev L., Niemi A.J., Hidden symmetry and duality in a charged two condensate Bose system, Phys. Rev. B, 2002, V.65, 100512, 4 pages; cond-mat/0106152.

[8] Fayzullaev B.A., Musakhanov M.M., Pak D.G., Siddikov M., Knot soliton in Weinberg-Salam model, Phys. Lett. B, 2005, V.609, V.442-448; hep-th/0412282.

[9] Faddeev L., Niemi A.J., Partially dual variables in SU(2) Yang-Mills theory, Phys. Rev. Lett., 1999, V.82, 1624-1627; hep-th/9807069.

[10] Langmann E., Niemi A.J., Towards a string representation of infrared $S U(2)$ Yang-Mills theory, Phys. Lett. B, 1999, V.463, 252-256; hep-th/9905147.

[11] Shabanov S.V., An effective action for monopoles and knot solitons in Yang-Mills theory, Phys. Lett. B, 1999, V.458, 322-330; hep-th/9903223.

[12] Cho Y.M., Lee H.W., Pak D.G., Faddeev-Niemi conjecture and effective action of QCD, Phys. Lett. B, 2002, V.525, 347-354; hep-th/0105198.

[13] Gies H., Wilsonian effective action for $S U(2)$ Yang-Mills theory with Cho-Faddeev-Niemi-Shabanov decomposition, Phys. Rev. D, 2001, V.63, 125023, 8 pages; hep-th/0102026.

[14] Dhar A., Shankar R., Wadia S.R., Nambu-Jona-Lasinio type effective Lagrangian. 2. Anomalies and nonlinear Lagrangian of low-energy, large N QCD, Phys. Rev. D, 1985, V.31, 3256-3267.

[15] Aitchison I., Fraser C., Tudor E., Zuk J., Failure of the derivative expansion for studying stability of the baryon as a chiral soliton, Phys. Lett. B, 1985, V.165, 162-166.

[16] Marleau L., Rivard J.F., A generating function for all orders skyrmions, Phys. Rev. D, 2001, V.63, 036007, 7 pages; hep-ph/0011052.

[17] Vakulenko A.F., Kapitanskii L.V., stability of solitons in $S^{2}$ in the nonlinear $\sigma$ model, Sov. Phys. Dokl., 1979, V.24, N 6, 433-434.

[18] Ward R.S., Hopf solitons on $S^{3}$ and $\mathbf{R}^{3}$, Nonlinearity, 1999, V.12, 241-246; hep-th/9811176.

[19] Manton N., Sutcliffe P., Topological solitons, Cambridge University Press, 2004.

[20] Pais A., Uhlenbeck G.E., On field theories with non-localized action, Phys. Rev., 1950, V.79, $145-165$.

[21] Smilga A.V., Benign versus Malicious ghosts in higher-derivative theories, Nucl. Phys. B, 2005, V.706, 598-614; hep-th/0407231.

[22] Eliezer D.A., Woodard R.P., The problem of nonlocality in string theory, Nucl. Phys. B, 1989, V.325, 389-469.

[23] Jaén X., Llosa L. Morina A., A reduction of order two for infinite order Lagrangians, Phys. Rev. D, 1986, V.34, 2302-2311.

[24] Simon J.Z., Higher derivative Lagrangians, nonlocality, problems and solutions, Phys. Rev. D, 1990, V.41, 3720-3733.

[25] Faddeev L., Niemi A.J., Wiedner U., Glueballs, closed fluxtubes, and eta(1440), Phys. Rev. D, 2004, V.70, 114033, 5 pages; hep-ph/0308240.

[26] Bae W.S., Cho Y.M., Park B.S., Reinterpretation of Skyrme theory, hep-th/0404181.

[27] Kondo K.-I., Magnetic condensation, Abelian dominance, and instability of Savvidy vacuum, Phys. Lett. B, 2004, V.600, 287-296; hep-th/0410024. 